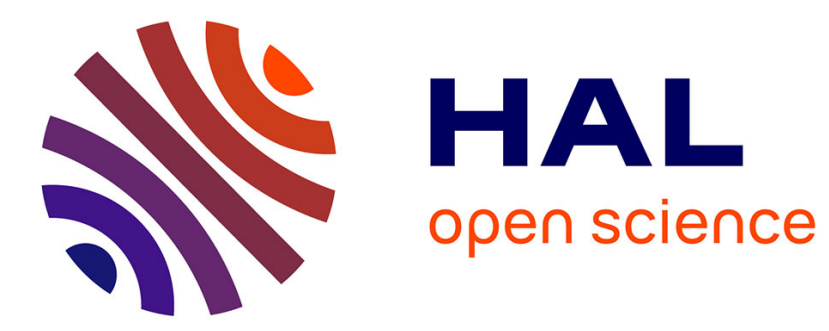

\title{
The adhesion of growing oxide scales to the substrate
}

G. Wood, J. Stringer

\section{To cite this version:}

G. Wood, J. Stringer. The adhesion of growing oxide scales to the substrate. Journal de Physique IV Proceedings, 1993, 03 (C9), pp.C9-65-C9-74. 10.1051/jp4:1993904 . jpa-00252334

\section{HAL Id: jpa-00252334 https://hal.science/jpa-00252334}

Submitted on 1 Jan 1993

HAL is a multi-disciplinary open access archive for the deposit and dissemination of scientific research documents, whether they are published or not. The documents may come from teaching and research institutions in France or abroad, or from public or private research centers.
L'archive ouverte pluridisciplinaire HAL, est destinée au dépôt et à la diffusion de documents scientifiques de niveau recherche, publiés ou non, émanant des établissements d'enseignement et de recherche français ou étrangers, des laboratoires publics ou privés. 


\title{
The adhesion of growing oxide scales to the substrate (*)
}

\author{
G.C. Wood $\left({ }^{1}\right)$ and J. Stringer $\left({ }^{2}\right)$
}

( $\left.{ }^{1}\right)$ Corrosion and Protection Centre, UMIST, PO Box 88, Manchester, M60 1QD, UK

$\left({ }^{2}\right)$ Generation and Storage Division, EPRI, 3412 Hillview Avenue, PO Box 10412, Palo Alto, California 94303, U.S.A.

\begin{abstract}
There is only limited new understanding of the structure of the evolving dynamic metal/oxide interface itself but there is valuable circumstantial evidence from cooled specimens and from other interfaces. Identification of flaws for crack initiation, definition of a composite defect and elaboration of delamination models, involving a plastically-relaxed crack tip region, have been undertaken. Loss of adhesion has received more attention, by the alternatives of film buckling or crack propagation along the metal/oxide interface by wedging, including cases where stress relief occurs by creep. Mapping of scale failure modes has progressed. Computation and modelling using electronic and atomistic models are useful, but "clean" metal/oxide and contaminated interfaces require in-depth work. Stress development and relaxation are complex. Models involve dislocation climb and the role of vacancy transport and cavity development. Sweeping of various particles by moving boundaries is receiving renewed analysis. The role of convoluted metal/oxide interfaces in promoting or diminishing adhesion has been analysed quantitatively. Explanation of reactive element effects by the embedded atom method is instructive, as are the relative roles of $S$ and $P$ in weakening interfacial adhesion.
\end{abstract}

\section{Introduction.}

The factors involved in oxide/metal separation, namely oxide/metal adhesion, stress generation, structure and evolution of the growing, dynamic oxide/metal interface, and the reactive element effect, were considered previously [1]. A longer paper on these aspects and approaches to improve adhesion is being prepared. The present paper highlights some recent literature and indicates where earlier research needs fresh emphasis.

Oxide/metal separation occurs either at the interface itself (adhesive failure) or near it (cohesive failure). There are significant differences in such processes with a dynamic growing oxide, because of the flux of material across the interface, through the substrate and the scale evolving a structure with a specific morphology, including specific grain structures and void distributions and affording stress relief processes akin to plastic deformation, compared with a static interface where this is largely absent.

Oxide adhesion to a metal substrate may be discussed formally in terms of electrostatic interactions associated with space charge development, chemical bonding across the interface, van der Waals forces and mechanical interactions related to non-planar interfaces. This must be consistent with the scale growth mechanism and the evolving metal/oxide interface.

Adhesion relates to interfacial energy, $\gamma_{i}$, through

$$
W_{\mathrm{ad}}=\gamma_{\mathrm{i}}-\gamma_{\mathrm{m}}-\gamma_{\mathrm{ox}}
$$

(*) Keynote lecture. 
where $W_{\mathrm{ad}}$ is the work of adhesion, and $\gamma_{\mathrm{m}}$ and $\gamma_{\mathrm{ox}}$ are the surface energies of the separated metal and oxide phases. Their magnitudes can affect the steady-state interface morphology, including development of interfacial voids. Practice is more complex, the total interfacial energy adjusting to stress development, evolution of non-planar interfaces, grain or phase boundaries and associated grooving. Interfaces vary from being essentially atomically complete, to regions of intimate contact separated by voidage or impurity segregation and, finally, where there is only physical contact at asperities.

Many failures are thus really cohesive in the oxide near the interface and involve oxide growth stresses, oxide and metal plasticity and thermal cycling effects due to differential thermal contraction. Local failures may involve cracks normal to the interface (through cracks), cracks parallel to the interface (delamination cracks) or spalling. Repeated oxide spalling is often life-limiting and is dangerous where the substrate becomes depleted in the preferentially oxidizing constituent (typically $\mathrm{Cr}, \mathrm{Al}$, or $\mathrm{Si}$ ) and breakaway oxidation ensues. Voids in oxidizing systems can play roles such as crack starters or stoppers and arise from vacancy transport and annihilation.

Adhesion between various coatings and equally diverse substrates is critical in materials studies and in surface engineering. Reviews [2,3] consider many general factors and some related to oxidation processes [3]. A comprehensive Workshop report [4] ignores the oxidizing metal case but is challenging and emphasizes the substantial theoretical and experimental developments needed. Possible in-situ spectroscopic and microscopic imaging of the interfacial zone and ultrafast techniques such as repetitive pulse heating, step exposure to gases, or shock wave exfoliation afford promise [5].

\section{Factors in the separation of the oxide from the metal.}

The origin and magnitude of flaws for crack initiation, and of void accretion, especially larger flaws required to initiate buckling and spalling, need definition [6]. Void growth and coalescence into cracks seem to require a normal interfacial tensile stress which exists on convex surfaces. The conditions for microcrack initiation in multiphase and single-phase, amorphous and fine-grained oxides are available [6].

The energy dissipated during bond rupture, with plasticity absent, is:

$$
G=\zeta\left(-W_{\mathrm{ad}}\right)
$$

where $G$ is the fracture energy and $\zeta=1-3$ for single crystal cleavage but much larger and dominant multipliers (20 or higher) are obtained for charged, separated surfaces, when plastic deformation occurs ahead of the crack tip (Fig. 1) and for non-planar interfaces, thus deflecting the crack [7]. The "plastic relaxation" of the residual stresses by fracture-created dislocations has been demonstrated experimentally. Metal-oxide interfaces at high temperature typically have a greater quasi-static fracture resistance than oxide unless there are voids, creep cracks, S segregation etc.

Oxide decohesion occurs when the strain energy in the oxide, due to differential thermal contraction on cooling, attains the fracture energy of the metal/oxide interface, treated empirically (Fig. 2) [8]. Decohesion under tensile stressing has been considered [6] but failure under compression is more important and has been derived mathematically for elastic or creep buckling (type II) and interfacial crack propagation (type I) [6, 8]. For film spalling by mechanism II (Fig. 2), the initial crack radius must satisfy buckling requirements and the strain energy release, $G$, due to buckling should exceed the fracture resistance, $G_{\mathrm{c}}$, of the film or interface. 
CRACK LOCATION



Fig. 1. - Adhesive failure in metal/ceramic systems (Adapted from Cannon [7 and allied publications]). Similar figures are available for cohesive failures $[1,7]$.

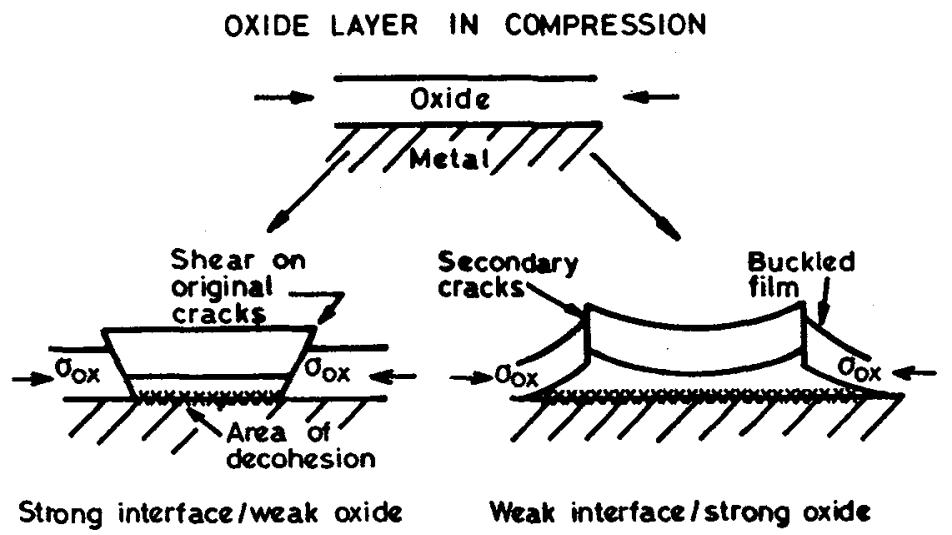

Fig. 2. - Schematic representation of cracking, decohesion blistering and spallation in oxide scales under compression (adapted from Evans H.E. et al. $[8,9]$ ).

Mechanism I holds where the intrinsic strength of the metal-oxide interface is high, involving no cohesive shear failure in the oxide, and occurs by tensile crack propagation through the $\mathrm{SiO}_{2}$ layer along the metal-oxide interface, aided by wedging action of the adjacent oxide/tensile/wedge crack (Fig. 2). Initially, this seems less likely but the mechanism has been extended by modelling spallation in $20 \mathrm{Cr} / 25 \mathrm{Ni} / \mathrm{Nb}$-stabilised steel, employing finite element analysis, using special elements to describe the metal/oxide interfacial region, where deformation is under either elastic or creep conditions [9]. Strain energy release in spallation computations indicated that the strain energy model held under alloy creep conditions. An "effective strain energy" is introduced, at least an order of magnitude greater than the true fracture energy. "Stable separation" at the interface rather than sudden "unstable decohesion" is also a possible fracture mechanism, which was discussed previously $[1,10]$.

A simple method exists [11] for determining the dimensions of a "composite defect" in scales by summing all the actual defects, using linear elastic analysis, and thus predicting inplant component failure. Fracture toughness is assessed and the stress intensity factor for growing surface oxide scales calculated. All partial defects are analyzed, involving shape, bending and membrane correction factors, and a single "composite defect" of defined length and thickness is determined by summing the effects of individual defects, using planar defect interaction rules. Fracture toughness for scales on various alloys and temperature and scale thickness effects have been calculated. 
Robertson and Manning [12] have considered flaws and used fracture mechanics to derive three modes of scale failure under rapid applied strains, where oxide and metal creep or local yield relaxation is allowed. Brittle spalling, extended scale adherence by substrate yield with cracked oxide islands "floating" on the metal and finally oxide ductility all occur. Figure 3 is a typical failure mode map as a function of strain and scale thickness. Strain rather than stress in used because strain is continuous at a thin scale/massive metal interface and is the observable quantity. Mapping is also usefully used by Schütze [10], who also points out the importance of flaw size in the scale rather than the scale thickness as a parameter.

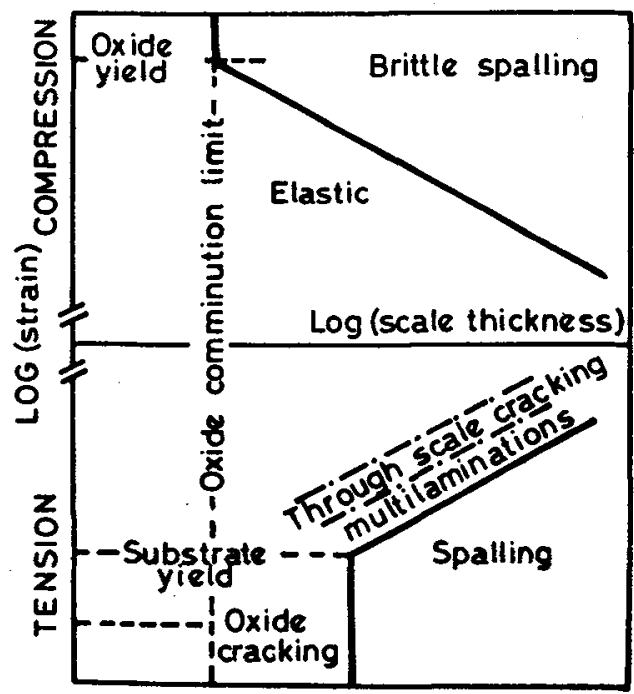

Fig. 3. - Failure mode map for tensile and compressive strains (after Robertson and Manning [12]).

$A b$ initio prediction of cracking and spalling trends is beyond present capability due to complex stress development, void formation and oxide damage [6]. Some aspects are well described by existing models. Experimental cracking observations should be classified into groups and predominant trends elucidated. Cannon and A.G. Evans have authored many other papers needing consideration in the oxidation area as the subject develops.

\section{Factors in oxide/metal adhesion.}

Important factors $[1,13]$ include the adhesive force when two surfaces are in sufficiently intimate contact (Stefan effect) and electrostatic forces, including van der Waals forces and Coulomb interactions arising from the electron transfer from one surface to another, by mechanisms including image force interactions. Stoneham and Tasker [14, 24] show that the scale/metal interface is intrinsically strong on an atomic scale and that the surface energy of the ideal oxide/metal interface is equal to that of the bulk oxide, as emphasized by Robertson and Manning [12]. The ionization transfer is a form of chemical bonding, and a second form, similar to covalent bonding, involves extended electron orbitals linking the 
two phases across the interface [15]. Thus the real bond may have some covalent and some charge transfer character.

Various static interfaces involve surprisingly similar adhesive considerations. Buckley $e t$ al. [4] point out that only the perfect, single crystal, pure metal-metal situation has so far been modelled as two surfaces are brought into contact, whereas experimental evidence is more general. When two solids are brought together adiabatically and reversibly at $T=0 \mathrm{~K}$, at each separation a between the two surfaces the total energy $E(a)$ is a minimum functional of the electron density and atomic coordinates. The basic adhesive energy $E_{\text {ad }}(a)$ is then

$$
E_{\mathrm{ad}}(a)=\frac{E(a)-E(\infty)}{2 A}
$$

where $A$ is the cross-sectional area. $E_{\text {ad }}(a)$ - separation processes have some universality for various types of film and interface but the effects of elastic, inelastic and plastic deformation, impurity interfacial defects, surface roughness and asperity effects then need evaluation for metallic, semiconducting and ceramic interfaces for comparison with experimental bond strength and yield stress data and fracture location.

Ionic and covalent bonding differences, and interdiffusion and chemical segregation effects, can be distinguished in metal-semiconductor models. Specific situations include: i) various metals contacting a single semiconductor, to correlate adhesion forces and interfacial chemical bond energy; ii) a single metal with various semiconductors (ionic and covalent) to establish relationships between bond type and adhesive strength. Regarding ceramic grain boundaries, bridging the gap between electronic structure calculations and centralforce atomistic simulation methods is important [4]. Electronic structure calculations give insight into the nature of interactions between atoms in interfaces, the effects of impurities etc. Realistic interfaces cannot be treated currently due to the many atoms involved. So the insight gained at electronic structure level needs to be transferred to the atomistic level where simulation techniques can handle thousands of atoms using supercomputers. Improved computers help but new theoretical methods beyond jellium surfaces, transition metal overlayers and semiconductor interfaces are required [4]. Calculations for metal-ceramic interfaces are more difficult. Existing models do not treat the dynamic metal-oxide interface.

Surface roughness, pegging, or convolution of metal/oxide interfaces can improve scale adhesion. A convoluted interface may deflect a propagating crack, with a significant effect on the fracture energy [16]. Fracture toughness is primarily due to crack-tip shielding from plastic stretching of metal-film ligaments bridging the interfacial crack in a zone behind the crack tip.

Oxide residual stresses depend on metal-oxide interface waviness and stress relaxation characteristics [17]. Geometry is likely to influence through-scale cracking produced by tensile stresses and buckling and spalling evident under compressive stressing. Analysis of a castellated model of a rough interface, in terms of roughness height and length, showed that roughness increased the critical energy release rate, $G_{\mathrm{IC}}$, by a factor of up to 10 , because of the energy needed to "unlock" each castellation [18]. Roughness increased the non-plastic part of $G_{\text {IC }}$. With pegged oxide, spalling may be diminished as the crack has to propagate through a metal/oxide composite. However, although metal plasticity may blunt interfacial cracks, it can be detrimental elsewhere because of residual stresses created around cracks.

For a smooth quasi-infinite plane surface, the interfacial stresses approach zero if the scale has no cracks or discontinuities [10]. Wavy or defective interfaces yield inhomogeneous deformation leading to local normal stresses, which produce or extend interfacial defects such as voids when they exceed critical values. The defect compositing method [11] can assess the 
effect of voids on $G_{I C}$. The interface can be weakened by voids and strengthened by roughness to exceed its intrinsic strength, helping understanding of pegs and their optimal size to improve adhesion.

"Pseudoplasticity", involving scale microcracking and rapid healing, is another factor involved in delaying or preventing oxide/metal separation [10].

\section{Stress generation in the oxide/metal system.}

The three main types of stress driving spalling are: stress caused by differential contraction of metal and oxide on cooling; interfacial stresses due to epitaxial strains and arising from adhesion; growth stresses developed isothermally $[1,13]$.

Pieraggi and Rapp [19] describe scale growth on metals by cation diffusion (e.g. NiO) in terms of intrinsic dislocations for an epitaxial metal/oxide interface. This is consistent with the observed high local deformation, intimate contact and epitaxial relations between inner oxide grains and substrate. Metal vacancy annihilation at the metal/oxide interface occurs by intrinsic misfit interface dislocations climbing into the metal. Epitaxy is maintained by adjustment of spacing of residual interface dislocations, thus generating tensile stress in the metal and compressive stress in the scale. Above a critical interfacial strain, dislocation glide in metal and scale recreate or resupply the interface dislocations, producing plastic deformation of metal and oxide close to the interface and maintaining epitaxy with the receding metal. Non-epitaxial behaviour awaits analysis.

The associated void development in the metal and any significance of "vacancy injection" have been reviewed. Thus growth of intergranular voids in metal substrates during oxidation is often due to induced tensile stress, causing void growth by Hull-Rimmer mechanisms [20, 21]. Vacancies are destroyed at the metal/oxide interface. Interfaces of inert, non-growing oxides are not normally good sinks for metal vacancies but can be if atomic movements in metal and oxide phases occur. Thus, diffusional properties of a metal-oxide interface change dramatically when oxidation stops. Cation vacancy injection into metal requires vacancies of low binding energy to an oxide/metal interface with widely spaced sinks [22]. Sub-surface cavities can also occur by local element depletion, $\mathrm{C}$ /oxide interaction, gas bubble formation, and Kirkendall-type diffusion processes [22]. Vacancies at the metal/oxide interface may annihilate at discrete interface sinks, surface migrate to a aggregate as cavities, or inject into the metal to give cavities. The fate of cavities is determined by the interface sink spacing and the intrinsic residence time of vacancies in the interface. Conditions for vacancy entry into metal from oxide are demanding but some authors still accept the concept.

The sign and magnitude of stress depend on interface coherency, oxide microstructure and thickness, surface curvature and diffusion parameters determining the nature and location of oxide growth, creep relaxation and geometrical discontinuities [6]. Trends in stress level and distribution are discussed by continuum stress analysis relating to amorphous and finegrained oxide, or by appraisal of the chemical potential gradients and atomic fluxes that govern stresses in columnar/large grained oxides.

\section{The structure of the metal/oxide interface and its evolution.}

Adhesion has been reviewed in terms of surface structure, chemistry and electrostatics [23]. Modelling of $\mathrm{NiO}$ oxide grain boundaries has not been extended to metal/oxide interfaces [24]. An early review of coherent, semi-coherent and incoherent interfaces and high angle 
boundaries, and their specific roles as transport paths and defect sinks and sources, warrants study [25]. The important morphological, compositional and structural evolution of the moving metal/oxide interface has been studied less.

The lowest energy situation for an adherent oxide is apparently an interface essentially planar at long range, which accommodates to the minimum energy planes of the oxide on a fine scale. This is moderated by oxide and metal grain boundaries, providing grain boundary grooving, local transport processes and stress effects [1]. Real interfaces can be very complex.

Of interest in understanding an evolving metal-oxide interface is whether or not a particle can "move" within a solid matrix and by what mechanisms. Solid and liquid internal oxide particles in copper can be dragged by migrating grain boundaries, with the particles accumulating on the boundary, leaving a particle-denuded volume behind [26]. The boundary speed is limited by the drag speed of the particles. Above $1000^{\circ} \mathrm{C}$, amorphous $\mathrm{SiO}_{2}, \mathrm{GeO}_{2}$ and $\mathrm{B}_{2} \mathrm{O}_{3}$ particles are relatively fluid, act like bubbles and are equally mobile, the mobility being limited by diffusion of copper atoms around the particle. At lower temperature, the particle mobility depends on its composition and viscosity and thus on the rate of transport or self-diffusion within the particle. Particle motion involves removal of copper atoms from in front of the particle and flow of particle atoms or molecules into the space. The second process controls the mobility of solid, or very viscous, particles. More solid particles, e.g. $\mathrm{SiO}_{2}$, are less mobile at lower temperature and their mobility is controlled by transport, or self-diffusion, within the particles. Crystalline $\mathrm{Al}_{2} \mathrm{O}_{3}$ particles show no measurable mobility in the matrix below the matrix melting point.

Gibbs and Harris [25], in discussing this work, conclude that semi-coherent $\mathrm{Al}_{2} \mathrm{O}_{3}$ particles are immobile in copper; mechanically entrapped incoherent $\mathrm{Al}_{2} \mathrm{O}_{3}$ particles are mobile; liquid $\mathrm{GeO}_{2}$ and $\mathrm{B}_{2} \mathrm{O}_{3}$ are mobile, with velocities consistent with interface diffusion at roughly the same rate as grain-boundary, self-diffusion in copper; grown-in particles of amorphous $\mathrm{SiO}_{2}$ have very low mobilities, controlled by mass transport within the particles.

These results are consistent with immobility in coherent or semi-coherent interfaces because the interfaces cannot act as defect sources or sinks. Mechanically-entrapped or liquid particles are under interface diffusion control. The low mobility of $\mathrm{SiO}_{2}$ particles relates to whether or not matrix lattice defects can be created or destroyed at the interface by rearrangement of only matrix atoms. If they can, high mobility controlled by interface diffusion of matrix material is anticipated. For strong "chemical bonding" across an interface, rearrangement of particle molecules occurs at interface sinks or sources of matrix defects. One possibility is simple local movement of particle molecules annihilating matrix defects, giving velocity controlled by an interface reaction when molecular mobility is low. The other is that particle and matrix lattice defects may be created or annihilated in pairs at the interface, giving volume or interface diffusion control of particle material.

Internal particles of $\mathrm{Al}_{2} \mathrm{O}_{3}, \mathrm{Cr}_{2} \mathrm{O}_{3}$ and the oxides of $\mathrm{V}$ are incorporated into growing $\mathrm{NiO}$ scales, on dilute nickel alloys, but oxides of $\mathrm{W}$ are only partly incorporated and oxides of Mo are not incorporated but are swept by the metal/oxide interface and essentially pile up [24]. There seems no obvious correlation with particle size, shape, solid or liquid condition, or conversion to other oxides before, during or after incorporation. Other factors such as the way particles influence relative transport processes within the oxide and at the metal/oxide interface are being considered.

Harris [21] raises numerous questions and answers some regarding the possible role of second-phase particles in inhibiting oxidation. Depending upon their efficacy as metal vacancy sinks or markers, their involvement in stress relief mechanisms and the direction of transport in the growing scale, they may congregate, pile up, get incorporated into the scale or cause a wavy interface to develop. Accumulation at the growing metal/oxide interface may 
affect its capability of perhaps acting like a high angle grain boundary, and being an infinite source and sink for vacancies.

Non-planar interfaces may be produced due to rate control by diffusion of the oxideforming element outwards in the alloy [28], as a consequence of multicomponent diffusion considerations causing two-phase oxide layers to develop, by viscous fingering, by growth stress effects causing deformation of the metal and by multiphase effects.

\section{The reactive element effect in relation to adhesion.}

The reactive element may affect the model of decohesion [8], where the stored elastic strain energy exceeds that required to generate new surfaces [29, 30], by causing: a) reduction in in-scale compressive stresses, which drive the failure; b) enhancement of the scale-substrate interfacial adhesion; or $\mathrm{c}$ ) improvement in oxide fracture toughness.

Modification of growth processes, by diffusion blocking in grain boundaries, could give thinner scales with insufficient thermal mismatch strain energy to cause spalling on cooling; change cation/anion contributions to scale growth, causing different types and distributions of growth stresses; reduce through scale cracking by increasing the required energy; reduce void formation and loss of interfacial contact by promoting oxygen rather than cation vacancy diffusion in; cause a discontinuous, even interlocking, $\mathrm{SiO}_{2}$ layer of varying crystallinity at the bottom of $\mathrm{Cr}_{2} \mathrm{O}_{3}$ scales, precluding a continuous fracture path.

Changes in scale microstructure might facilitate plastic deformation or microcracking and relax stored energy by increased creep or grain boundary sliding, depending on grain size and shape changes. Enhanced mechanical strength and fracture toughness of the scale, or RE particles arresting cracks developing during stress scale, or RE particles arresting cracks developing during stress relief, might inhibit failure by increasing the energy for through crack initiation and propagation. Large RE particles might, conversely, act as stress concentrators or crack initiators, producing the opposite effect. Comparisons at constant scale thickness are required. Improved scale/substrate interfacial adhesion is also possible.

Peg formation is not generally required for RE improved adhesion (e.g. with coated and ion implanted alloys) and some coarse pegs make matters worse by initiating crack propagation and affecting adhesion/cohesion adversely. Sometimes, micropegs may enhance adhesion by causing intimate alloy/scale contact; act as a mechanical graded seal, with the mixed alloy/oxide region gradually accommodating the macroscopic mismatch in specific volumes and expansion coefficients; improve fracture toughness by pegs increasing path lengths for interfacial crack propagation, modifying total stress fields and increasing the capacity for energy dissipation by plastic alloy protrusions.

The vacancy sink effect may still have some consequences [29]. $\mathrm{As}_{\mathrm{Cr}_{2}} \mathrm{O}_{3}$ and $\mathrm{Al}_{2} \mathrm{O}_{3}$ dispersions promote adhesion with $\mathrm{Cr}_{2} \mathrm{O}_{3}$-formers and $\mathrm{Al}_{2} \mathrm{O}_{3}$-formers, enhanced chemical bonding is not required but may occur by direct effect or by influencing grain size and texture of alloy or oxide to enhance interfacial matching.

Bennett and Moon [30] subsequently claim that impurity gettering, keying/pegging, chemical bonding and scale plasticity cannot be critical for their $\mathrm{Cr}_{2} \mathrm{O}_{3}$-formers. The thinner scale with insufficient strain energy to initiate spalling argument may be important [6], and also a reduction in number of scale or interfacial flaws.

Dearnaley [31] considers the adhesion strength between oxide and metal using Cottrell's [32] recent analysis of grain-boundary weakening employing the embedded atom method. The electron gas of the metal host rearranges itself to accommodate an embedded atom and the resulting interaction energy is calculated relative to the energy level of the free atom. A 
foreign atom may weaken or strengthen a grain boundary depending on its tendency to segregate at grain boundaries, the extent to which interaction can be expressed as an embedding energy (or in terms of covalent bonding with the host atom) or, for substitutional impurities displacing host atoms, the relative cohesive energies of the two elements.

$\mathrm{S}$ is a powerful and $\mathrm{P}$ a moderate weakener of iron.

Electronegative atoms repulse the electron gas of the host metal and weaken the boundary. B and C, covalently bonded to iron, act as strengtheners. Segregated atom interaction can neutralise these effects.

The metal-oxide interface is like an extended grain boundary, or series of boundaries, especially when outward cation diffusion predominates. Impurities affect the boundary strength. The thermal energy drives impurity segregation at the alloy-scale interface and individual defects contribute to trapping mechanisms. For impurity segregation at a surface, there is an electronic contribution to the heat of segregation related to the surface core level shift, namely the difference in core electron binding energy between surface and bulk atoms.

The strong affinity of $\mathrm{Y}$ for oxygen satisfies Cottrell's third criterion in an oxygen-rich environment, by forming strongly-bonded species at the interface. Interfacial strengthening also occurs between scale oxide grains. Yttrium may also lessen the $\mathrm{S}$ weakening effect, giving $\mathrm{Y}_{2} \mathrm{~S}_{3}$; the strong electronegativity difference contributes to the thermodynamic stability of yttrium sulphides. This eliminates weakening caused by $S$ and provide potential strengthening. Beyond a critical S coverage of 0.25 , S could inhibit $\mathrm{C}$ segregation in Co, perhaps by preferential site occupancy. Thus, a further weakening at grain boundaries and interfaces by a strongly-segregated weakener like $S$ may be by competition with potentially strengthening species like $\mathrm{N} \& \mathrm{C}$.

Ikeda [33] reported that, although $\mathrm{S}$ segregated at the interface caused $\mathrm{Al}_{2} \mathrm{O}_{3}$ spalling, $\mathrm{P}$ segregation there had no detrimental effect. Reactive metal elements suppressed $\mathrm{S}$ segregation but not $\mathrm{P}$. $\mathrm{Y}_{2} \mathrm{O}_{3}$ suppressed $\mathrm{S}$ and $\mathrm{P}$. This is because reactive metal elements give a stable sulphide and $\mathrm{S}$ is trapped as such in the matrix but phosphides are not as stable and decompose, liberating $\mathrm{P}$ at high temperature. However, $\mathrm{Y}_{2} \mathrm{O}_{3}$ suppresses surface segregation of impurity elements by adsorbing them at the $\mathrm{Y}_{2} \mathrm{O}_{3} /$ matrix interface. Because $\mathrm{Y}_{2} \mathrm{O}_{3}$ particles are highly curved, the interfacial energy is greater at the dispersed $\mathrm{Y}_{2} \mathrm{O}_{3} /$ matrix interface than at the $\mathrm{Al}_{2} \mathrm{O}_{3}$ layer/matrix interface. Thus $\mathrm{P}$ is considered preferentially trapped at the $\mathrm{Y}_{2} \mathrm{O}_{3} /$ matrix interface. So, $\mathrm{P}$ segregates at the surface without forming a phosphide film, whereas $\mathrm{S}$ is trapped as a sulphide in the matrix.

\section{Conclusions.}

1) Considerable progress is being made in understanding the general nature of static interfaces and the fracture mechanics related to loss of adhesion.

2) Quantitative modelling of various types of static interface, using computer methods, is now feasible at least for simple interfaces.

3) Much less progress is being made in understanding the evolution, maintenance and loss of adhesion of dynamic growing metal/oxide interfaces.

4) The significance of the degree of coherence of interfaces and of the interaction between sweeping interfaces and particles needs consideration. 


\section{References}

[1] Stringer J., Wood G.C., Mater. Res. Soc. Symp. Proc. 119 (1988) 185.

[2] Doerner M.F., Nix W.D., Crit. Reu. Solid State Mater. Sci. 14 (3) (1988) 225.

[3] International Workshop on "New Fundamentals of Scale Growth" P. Kofstad, A. Rahmel, R.A. Rapp, D.L. Douglass Eds. (Irsee, 1988) Oxid. Met. 32 (1989) 125.

[4] Panel Report on Interfacial Bonding and Adhesion, J.R. Smith Ed., Mater. Sci. Eng. 83 (1986) 169.

[5] KING E.W., Private communication.

[6] Evans A.G., Cannon R.M., Materials Science Forum, Trans. Tech. Publications, 43 (Switzerland, 1989) p. 243.

[7] CANnOn R.M., Am. Ceram. Soc. Bull. 64 (1985) 449.

[8] EVANS H.E., Mater. Sci. Tech. 4 (1988) 415.

[9] EVANS H.E., Mitchell G.P., LobB R.C., OWEN D.R.J., Nuclear Electric Report, Technology Division TD/FCB/REP/0070.

[10] SCHUTZE M., Mater. Sci. Tech. 4 (1988) 407; 6 (1990) 32.

[11] HANCOCK P., NICHOLlS J.R., Mater. Sci. Tech. 4 (1988) 398.

[12] Robertson J., MANNing M.I., Mater. Sci. Tech. 6 (1990) 81.

[13] STRINGER J., Werkst. Korros. 23 (1972) 747.

[14] Stoneham A.M., TASKeR P.W., Mater. Sci. Tech. 4 (1988) 382.

[15] ANderson A.B., MaHEndRu S.P., Smialek J.L., J. Electrochem. Soc. 132 (1985) 1695.

[16] OH T.S., Rödel J., CANnON R.M., Ritchie R.O., Acta Metall. 36 (1988) 2083.

[17] Evans A.G., Grumley G.B., Demaray R.E., Oxid. Met. 20 (1983) 193.

[18] Evans A.G., Hutchinson J.W., Acta Metall. 37 (1989) 909.

[19] PieragGi B., RaPP R.A., Acta Metall. 36 (1988) 1281.

[20] HARris J.E., Acta Metall. 26 (1978) 1033.

[21] HARRIS J.E., Mat. Sci. Tech. 4 (1988) 457.

[22] Evans H.E., Mat. Sci. Tech. 4 (1989) 1089.

[23] STONEHAM A.M., TASKer P.W., Ceramic Microstructures 86: Role of Interfaces, J.A. Pask Ed. (Plenum, New York, 1988).

[24] Duffy D.M., Tasker R.W., Physica B 131 B (1985) 46.

[25] Gibbs G.B., Harris J.E., Proc. Int. Conf. Interfaces, R.C. Gifkins Ed. (Butterworth's, London, 1969).

[26] ASHBy M.F., CenTAMORE G.M.E., Acta Metall. 16 (1968) 1081.

[27] Strawbridge A., StotT F.H., Wood G.C., Corros. Sci., in press.

[28] WaGner C., J. Electrochem. Soc. 103 (1956) 371.

[29] MOON D.P., AERE R12930 (January, 1988).

[30] BEnNeTt M.J., MOON D.P., AERE R13368 (January, 1989).

[31] Dearnaley G., Corros. Sci. 32 (1990) 113.

[32] CotTRell. A.H., Mater. Sci. Tech. 6 (1990) 326.

[33] IKEDA Y., TOSA M., YOSHIHARA Y., NII K., ISIJ Int. 29 (1989) 966. 